\title{
Design and Implementation of Linear Transformation Device for Current Frequency Isolation
}

\author{
Y. Wu \\ Northeast Dianli University \\ Jilin JILIN, China \\ G.N. Xu \\ Northeast Dianli University \\ Jilin JILIN, China
}

W.H. Gou

Jilin Dongjie Science and Technology Development Co., Ltd Jilin JILIN, China

\begin{abstract}
There are many problems such as various unknown signals, poor signals' stability and high error rates in the process of transmission. In view of the complex sensor distribution as well as various types of signals. This paper put forward a kind of device which can realize linear transformation from frequency signals to the frequency of the current linear transformation isolation. The device's circuit structure has a strong anti-interference ability, which can be applied to all kinds of sensor signal transmission system and has a great value.
\end{abstract}

Keywords-frequency; current; isolation linear transformation

\section{INTRODUCTION}

As an important part of a remote monitoring and intelligent system, sensors play a role in PC data acquisition, data analysis, and behavioral decision to provide stable and reliable real-time data. Aiming at solving the problems existed in complex sensor distribution and various confusing signals; this paper offers a solution to put forward a kind of device that can realize linear transformation frequency signals for the frequency of the current signal isolation linear transform current. Signal types have different sensor signals when it works, which can be united by a conversion device to a variety of sensor output signals to a standard reentry after current signals acquisition[1-2].

\section{THE HARDWARE STRUCTURE}

Current frequency conversion device can collect the standard $200-1000 \mathrm{~Hz}$ frequency signal, and converts it into a standard 4-20 ma current signal. As what shown in Fig.1 is a linear transformation frequency isolation current device of the overall circuit, which is made up from five parts, namely, the frequency signal input, photoelectric isolating circuit, frequency voltage conversion circuit, voltage conversion circuit, and current signal output terminal [3-4].

\author{
Y.F. Zhang \\ Jilin Dongjie Science and Technology Development Co., Ltd \\ Jilin JILIN, China \\ X.D. Wang \\ Jilin Dongjie Science and Technology Development Co., Ltd \\ Jilin JILIN, China
}

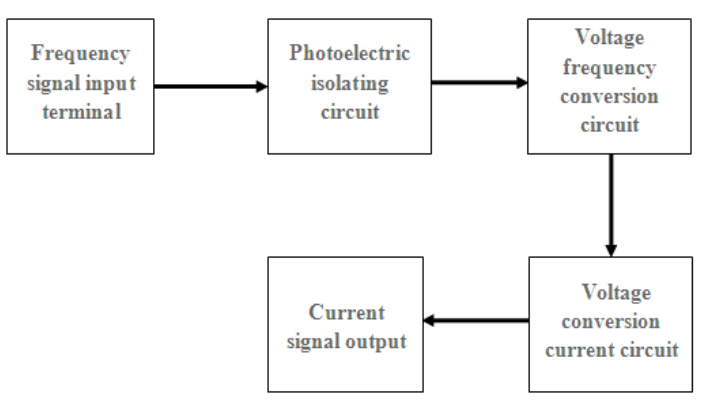

FIGURE I. FREQUENCY ISOLATION CIRCUIT DIAGRAM OF THE LINEAR TRANSFORM CURRENT MECHANISM.

\section{IIITHE WORKING PROCESS}

Current frequency conversion device working process is: 200-1000 Hz frequency signal input. The working process is to start with the photoelectric coupler photoelectric isolation, and then the photoelectric coupler circuit for the narrow pulse frequency signal output voltage to frequency conversion circuit. Frequency converter will pass frequency voltage conversion circuit by pressure adjustable potentiometer devices such as linear transform voltage signal to the voltage current circuit. Current voltage conversion circuit converts voltage is transformed linearly to 4-20 ma current signals, and finally through the current signal output it will be 4-20 ma current signals for long distance transmission. Fig. 2 shows the circuit principle diagram of a complete frequency conversion device [5-6].

As a standard 200-1000 Hz frequency input signal, it is converted into a narrow pulse frequency signal through the photoelectric coupler by a electrical isolation frequency signal, so it can transform sensors and devices effectively and do an electrical isolation, which can improve the anti-interference ability and guarantee the stability. 
There are complex industrial field environment, electromagnetic interference, such as environmental temperature variation and noises. Those factors make the poor performance of the chip and other electronic devices, so conversion precision and linearity is an important part of the design, whose hardware circuit is adopted to regulate conversion as well as frequency voltage conversion. And then by the change of output voltage into current, its electric performance is stable with excellent conversion linearity and a measurement precision.

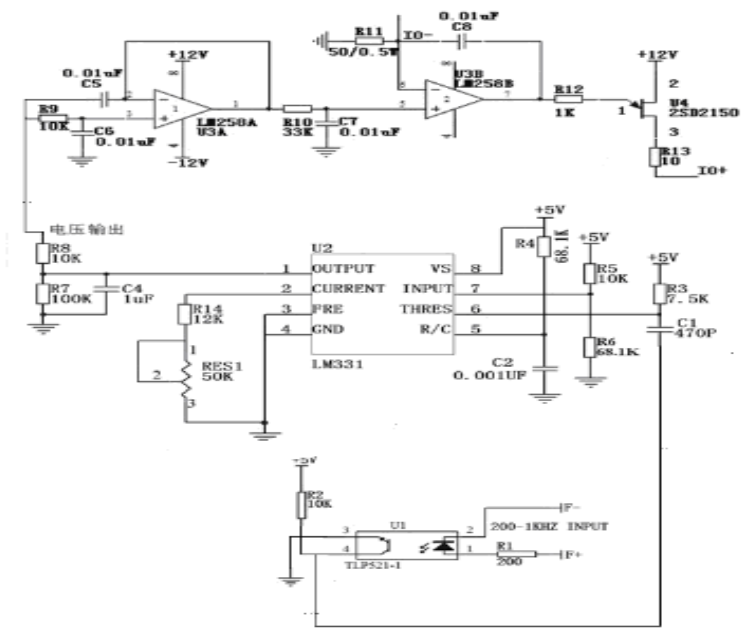

FIGURE II. THE CIRCUIT PRINCIPLE DIAGRAM OF A COMPLETE FREQUENCY CONVERSION DEVICE.

Current frequency conversion device whose components are commercially available mature products with high reliability. Among them: light electric coupler is Toshiba photocoupler TLP521-1; Pressure frequency converter U2's model is chip LM331 voltage frequency, and the model of operational amplifier is LM258 produced by TI company Texas instruments; Triode U4 model is 2 sd2150 produced by Germany's infineon[7-8].

\section{IVTEST RESULTS}

Adding $100 \mathrm{ohm}$ resistor load into device output and 200-1000hz standard signal into device input, we test input and output corresponding linear relationship. When the $200 \mathrm{hz}$ and $400 \mathrm{hz}, 600 \mathrm{hz}$ and $800 \mathrm{hz}$ and $1000 \mathrm{hz}$ standard signal input are being accessed, the voltage corresponding relationship is as follows:

When the input is 200hz,the output is $4 \mathrm{ma}$, and the resistance load is 100 ohms precise, the output voltage is $400 \mathrm{mv}$,oscilloscope sampling voltage is as shown is fig.3(a):

When the input is $400 \mathrm{hz}$, the output is $8 \mathrm{ma}$, and the resistance load is 100 ohms precise, the output voltage is $800 \mathrm{mv}$,oscilloscope sampling voltage is as shown is fig.3(b):

When the input is $600 \mathrm{hz}$, the output is $12 \mathrm{ma}$, and the resistance load is 100 ohms precise ,the output voltage is $1.20 \mathrm{v}$,oscilloscope sampling voltage is as shown is fig.3(c):
When the input is $800 \mathrm{hz}$,the output is $16 \mathrm{ma}$, and the resistance load is 100 ohms precise ,the output voltage is $1.60 \mathrm{v}$,oscilloscope sampling voltage is as shown is fig.3(d):

When the input is1000hz,the output is 20ma, and the resistance load is 100 ohms precise ,the output voltage is $2.00 \mathrm{v}$,oscilloscope sampling voltage is as shown is fig.3(e):

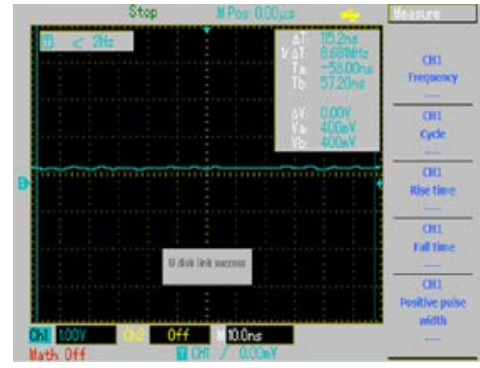

(a)

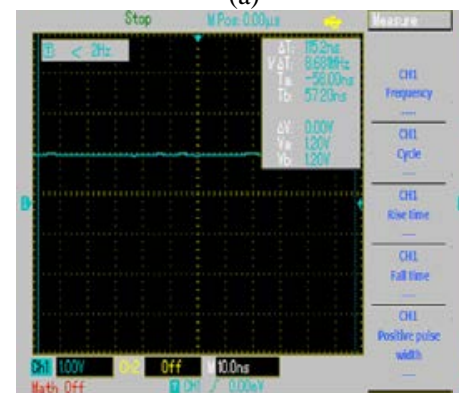

(c)

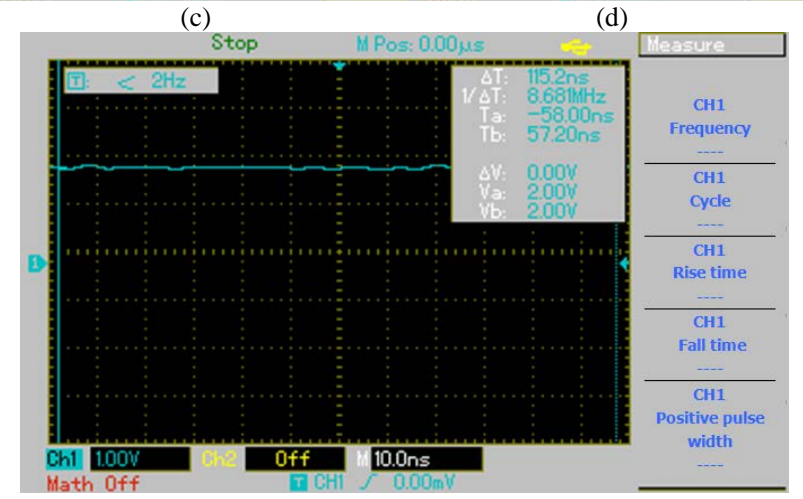

(e)

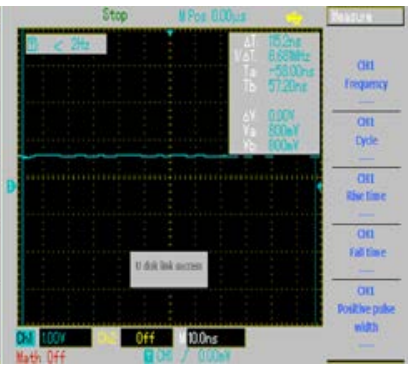

(b)

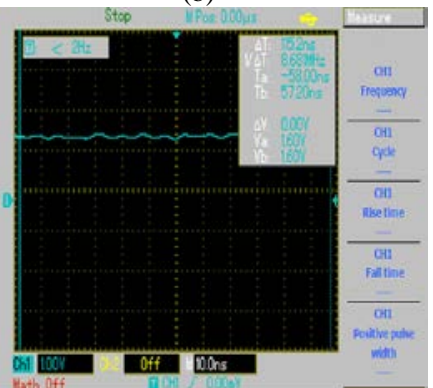

(d)
FIGURE III. OSCILLOSCOPE SAMPLING VOLTAGE.

As shown in Tab. 1, in the same field environment test the conversion linearity is up to $0.5 \%$ with the precision 0.2 on the Richter scale. Experiments show that the conversion device stability is good and it is suitable for different environment.

TABLE I. FREQUENCY CONVERSION CURRENT TEST DATA.

\begin{tabular}{|l|l|l|l|l|l|}
\hline $\begin{array}{l}\text { Frequency(Hz } \\
\text { ( }\end{array}$ & 200 & 400 & 600 & 800 & 1000 \\
\hline Current(Ma) & $\begin{array}{l}4.00 \\
4\end{array}$ & $\begin{array}{l}8.00 \\
6\end{array}$ & $\begin{array}{l}12.00 \\
8\end{array}$ & $\begin{array}{l}16.00 \\
9\end{array}$ & $\begin{array}{l}20.00 \\
8\end{array}$ \\
\hline
\end{tabular}

\section{SUMMARY}

The proposed frequency isolation linear conversion circuit of current device structure is reasonable with its low cost, small volume and strong anti-interference ability, which can achieve 
200-1000 Hz frequency signal linear transformation for 4-20 ma current signal. This can be widely used in electric power, remote monitoring, industrial control, instrumentation as well as medical equipment industry such as sensor signal transmission system with its great value.

\section{ACKNOWLEDGEMENT}

This work was supported by "Twelfth five-year" science and technology research project of jilin province department of education (Mine electrical and mechanical equipment of real-time monitoring and security early warning platform for research.) Project development plan of science and technology of Jilin Province (No.20140204049GX), the Science and Technology Planning projects of Jilin City (No.201434008).

\section{REFERENCES}

[1] Y.M. Chen and R.G. Cheng, Monolithic integrated TC9400 V/F and F/V converter, International Electronic Elements, 1997(2), pp. 31-35.

[2] L. Zhang, The Research of Prospective Analog Signals Isolation,University of Electronic Science and Technology of China,cheng du, 2005, pp. 1-5.

[3] P. Yang, J.C. Ding and L. Zhang, Design of An Isolated F\I Converter, Industry and Mine Automation, 2013, 39(1), pp. 113-115.

[4] Z.Y. Qu, S.L. Hou et al. ,Realization of Substation Visualization Training Platform, Journal of Northeast Dianli University, 2014, 34(3), pp. 75-79.

[5] R.X. Zhai, The principle and application of VFC320 pressure frequency converter, Application of Electronic Technique, 1998(12), pp. 44-46.

[6] L.P. He and J.Y. Li, DC Voltage Isolation Converting Circuit Design Based on HCNR200, Journal of Linyi Normal University,2009 ,31(6), pp. 43-46.

[7] G.L. Zheng, X.J. Ni and A.X. Yan, J.F. Zhao, High Isolated DC Voltage Detecting Technique Based on Linear Optocoupler ,Electrotechnics Electric, 2009(11), pp. 53-55.

[8] Y. Wu, Y.T. Zhou, T. Zuo and X.D. Wang, Based on the design and implementation of RFID electronic seal, Journal of Northeast Dianli University,2013(6), pp. 61-64. 